\title{
Precuing benefits for color and location in a visual search task
}

\author{
ESTHER VierCK AND JefF MiLler \\ University of Otago, Dunedin, New Zealand
}

\begin{abstract}
Selection in multiple-item displays has been shown to benefit immensely from advance knowledge of target location (e.g., Henderson, 1991), leading to the suggestion that location is completely dominant in visual selective attention (e.g., Tsal \& Lavie, 1993). Recently, direct selection by color has been reported in displays in which location does not vary (Vierck \& Miller, 2005). The present experiment investigated the possibility of independent selection by color in a task with multiple-item displays and location precues in order to see whether color is also used for selection even when target location does vary and supposedly dominant location precues can be used. Precues provided independent information about the location and color of a target, and each type of precue could be either valid or invalid. The precues were followed by brief displays of six letters in six different colors, and participants had to discriminate the case of a prespecified target letter (e.g., R vs. r). Performance was much better when location cues were valid than when they were invalid, confirming the large advantage associated with valid advance location information. Performance was also better with valid advance color information, however, both when location cues were valid and when they were invalid. But these color benefits were dependent on the closeness of the colored letter to the cued location. Our results thus suggest that selection by color in a multiple-item display, where location and color information are independent from each other and equalized, is mediated by location information.
\end{abstract}

Advance knowledge of the location of a target item has been shown to be helpful, not only when an item needs to be selected for further processing among a number of other items, but even when no distractor items are present (e.g., Posner, Snyder, \& Davidson, 1980). This effect has frequently been investigated within precuing paradigms. For example, Henderson (1991) asked participants to discriminate whether the letter $\mathrm{X}$ or $\mathrm{O}$ had been presented. The letter could appear in one of eight positions arranged in a circular fashion around fixation. At the beginning of each trial, the location of the target letter was cued. Cues could be valid, neutral, or invalid. Valid cues led to a performance advantage for both accuracy and reaction time (RT) measurements, when compared with the neutral cue condition. In addition, a comparison between invalid and neutral location cue trials revealed the presence of processing costs when misleading information was provided. These results indicate that a clear benefit is associated with location preknowledge in discrimination tasks.

\section{Evidence for Location Dominance}

Similar benefits of advance location information have been reported repeatedly in many different paradigms (e.g., Cheal \& Gregory, 1997; Posner et al., 1980; Theeuwes, 1989). Indeed, the findings are so robust that they have led some researchers to conclude that location is special and that selection of an item for further processing is virtually always mediated by location (Brawn \& Snowden, 1999; Cave \& Pashler, 1995; Garner, 1987; Kim \& Cave, 2001; Moore \& Egeth, 1998; Nissen, 1985; Shih \& Sperling, 1996; Tsal \& Lamy, 2000; Tsal \& Lavie, 1988, 1993). Accordingly, it has been controversial whether the observed advantages of advance color information (e.g., Kingstone, 1992) were due to a direct effect of color on selection or were mediated by location as the only feature allowing direct selection (e.g., Hübner \& Backer, 1999). The location-is-special viewpoint would accredit these advantages to the use of color to guide attention to the location of an item, where selection then takes place. In contrast, it is also possible that advance color information can be used for direct selection as is advance location information but that its effect has been overshadowed by the benefits observed for location information.

There is much evidence in support of this location-isspecial viewpoint (e.g., Garner, 1987; Lamy \& Tsal, 2000; Nissen, 1985; Tsal \& Lavie, 1993). Nissen, for example, used a multiple-item display followed by masks. In one condition, she cued an item by location; in another condition, by color. In each condition, participants had to give two responses. In the location cue condition, they were to report the color and shape of the item in the cued location, whereas in the color cue condition, they were to report the location and shape of the item in the cued color. The responses showed that for the location cues, the accuracy 
of color reports did not depend on reports for shape, indicating that, with location knowledge, the features of color and shape were processed independently. But this pattern was not observed for color cues. In that condition, the accuracy of shape responses was higher when location reports were correct than when they were incorrect; that is, the correct judgment of shape depended on the accuracy of the location judgment. On the other hand, the accuracy of location judgments did not depend on the accuracy of shape reports in this condition. The author concluded that location mediates selection in a situation in which the selection and response features differ. These findings have been reexamined by Monheit and Johnston (1994), however, in a study in which larger sample sizes were used and the effects of guessing were considered. In this study, the independence of color and shape was not replicated with location cues. Rather, it seemed that participants attended to a single location and reported both color and form correctly if that location was the relevant one. These results suggest that the observed dependency stemmed from the allocation of spatial attention, thereby strengthening the selection-by-location view even more.

Another study by Tsal and Lavie (1993) also supports the idea of location dominance over other features. Targets in their design could appear at one of two possible locations. At the beginning of each trial, small dots appeared next to these locations; one of these dots was colored. The color of this dot indicated whether the subsequently displayed letter in the left or the right position had to be reported. Although the location of the dot itself was irrelevant, trials in which the color dot was next to the location of the target led to faster responses than did trials in which the color dot was farther away. The authors argued that the additional time was needed in trials in which the target was presented farther from the dot because the participants had to redirect attention from the location of the color dot to the location of the target. Color was not beneficial in its own right; rather, it was linked to the location of its presentation.

In a recent study, Grabbe and Pratt (2004) examined whether location information and color information are functionally equivalent. Their display consisted of nine letters arranged in a circular fashion. The letters were displayed in three different colors, but letters with the same color were never adjacent to each other. The participants had to name a letter in a certain color and certain part of the display first, followed by as many other letters as possible from the display. Although color was the first selection criterion, the participants named many more letters adjacent to the initially named letter than letters with the same color. The authors concluded that location is special, in that it is the dimension automatically used in selection.

The experiments reviewed so far suggest a special status for location in selection, but color may still be used to directly select items for further processing under certain circumstances. In Nissen's (1985) experiment, participants had to focus on three dimensions of a target: location, color, and form. In both cuing conditions, location was part of the task and had to be focused on in order to fulfill the task requirements. It is possible that location information is the preferred selection feature whenever it is present and that an emphasis on location led the participants to select by location per se and, therefore, to use color cues only as a guide to the location of the target, since identification of location was always required. It is still possible, then, that participants may be able to use color for direct selection under conditions with less emphasis on location. A similar argument can be put forward for Tsal and Lavie's (1993) experiment. They used color simply as a location cue. It was therefore necessary to translate the meaning of the color into the associated location. Although color played a part, the main emphasis of the task was on selection by location. This may have led the participants to focus mainly on location and to neglect the color information. Thus, their results also do not completely exclude the possibility that direct selection by color could occur in a task with less focus on location. Similarly, in Grabbe and Pratt's (2004) experiment, the initial instruction to select a letter in a certain area may have biased the participants enough to give priority to location as a selection cue. Thus, location may not be the only feature suitable for direct selection, because previous research cannot completely exclude the possibility of direct selection by color.

\section{The Role of Color in Selection}

Color has clearly been found to be helpful when it defines a feature of the target in search displays. For example, Egeth, Virzi, and Garbart (1984) asked participants to search for a red $\mathrm{O}$ in a multiple item display. They manipulated the total number of items per display but kept the number of red items constant throughout. The authors concluded that the participants could limit their search to the subset of items defined by the red color. These results have been extended by Kaptein, Theeuwes, and van der Heijden (1995), who kept total display size constant but varied the number of elements that shared the target color. RTs increased with the number of items displayed in the target color. In a similar fashion, color has been used successfully as a defining element in conjunction searches to explore the role of different ratios between different types of distractors (Poisson \& Wilkinson, 1992; Shen, Reingold, \& Pomplun, 2000; Sobel \& Cave, 2002; Zohary $\&$ Hochstein, 1989). Together, these studies demonstrate advantages of advance color knowledge when color is part of the target definition. Although this line of research suggests a selection benefit for color, it remains unclear whether the color advantage is due to mediation by location or to direct selection by color.

Indeed, some studies in which paradigms with multiple stimuli displayed at the same time (i.e., when location varied) were used have implied that there is a more direct role of color in selection (Gold \& Pratt, 2001; Humphreys, 1981; Lambert \& Corban, 1992). For example, Lambert and Corban investigated the effects of combined expectations for color and location. The target item appeared either above or below fixation and had to be judged according to its orientation. It could be presented in either red or green. Each color was associated with a likely target location; for example, green items were more likely to be displayed 
above fixation $(p=.8)$. At the beginning of each trial, the participants were informed about the likely color of the target item. After the cue, an actual target was presented. It could be displayed in an expected or an unexpected color, and it could be displayed in an expected or an unexpected location. Not surprisingly, performance was best for targets presented in the expected location and color. In trials in which the target was displayed in the unexpected color, performance was better for targets in the location associated with the unexpected color than for targets in the location that was associated with the expected color. This result is inconsistent with the view that location is the dominant feature in selection. If location is more important than other features, color should have led to the associated location and, as a consequence, performance should have been better for items at this location, regardless of color.

Lambert and Corban's (1992) results imply an independent role for color during selection. Humphreys (1981) and Gold and Pratt (2001) also have provided evidence for this conclusion. Humphreys, for example, asked participants to judge the orientation of a target form. This target was always presented in the same location, so advance location information was always available. Only one distractor item was presented in a position next to the target. This distractor either changed its orientation randomly or was kept constant throughout the block. As a second manipulation, the target and the distractor were displayed in either the same or different colors. In the case of different colors, both target and distractor colors were kept constant for the whole block. If location is special, knowing the location of the target should be enough to ensure optimal performance, and color should not have any effect. Instead, color did influence performance. When the two forms were displayed in one color, performance was better in blocks in which the distractor orientation was constant than in blocks in which this orientation changed randomly. But when the items were displayed in different colors, performance did not depend on the consistency of distractor orientation. Thus, a target/distractor color distinction aided attentional selection even when location information was, in principle, sufficient to select the target. Humphreys also investigated whether participants could adjust their strategies according to the usefulness of the color information. In one condition, the target was always displayed in a certain color $(100 \%$ predictable); in a second condition, the target was usually presented in a certain color ( $75 \%$ predictable); and in a third condition, the target was equally likely to be in one of two colors ( $50 \%$ predictable). He found that performance was better when the target color was more predictable, and he concluded that participants could attend independently to either color or location, depending on the task requirements (i.e., color probability). Thus, these results indicate that selection can benefit from color information even when location information is clear, and they were interpreted by Humphreys as evidence that color and location features are equally beneficial in selection.

Although the experimental findings just reviewed cast doubt upon the location-is-special viewpoint by suggesting that color can sometimes be used directly without mediation by location, alternative explanations of all of these findings are possible. In Humphreys's (1981) experiment, the effect of color may have been caused by the spatial closeness of the items in the display. Distractor and target items were each $0.25^{\circ}$ below or above fixation. This closeness could have caused spatial confusions, even though the target location was known. If so, color may have functioned as an additional marker to support selection by location. The same could be true for Lambert and Corban's (1992) results.

In summary, studies conducted to determine the role of advance color information in multiple-item displays where selection takes place in space and location information is, therefore, potentially useful have led to contradictory conclusions. Location may dominate color, in the sense that whenever advance location information is provided, it becomes the only important feature in selection. On the other hand, color may have a direct effect in the selection process, independently of location.

\section{Evidence for Direct Selection by Color}

To investigate a potentially direct selection by color, Vierck and Miller (2005) conducted an experiment in which location was rendered useless. They used a rapid serial visual presentation (RSVP) paradigm experiment in which location did not vary. In this type of task, items are displayed one after another in rapid succession, often 15 items/sec. Vierck and Miller (2005) presented 15 colored letters in their upper- and lowercase versions. Participants were asked to discriminate the case of a prespecified target letter (e.g., H vs. h). At the beginning of each trial, a valid, neutral, or invalid color cue was provided. In valid trials, only the target letter was displayed in the cued color. In neutral trials, none of the letters - not even the target - was in the cued color. In invalid trials, one of the distractor letters was in the cued color, but the target was not. This design is analogous to that used in many location-cuing paradigms (e.g., Posner et al., 1980), apart from the fact that selection takes place in time, instead of space, in RSVP paradigms. Because location does not vary in an RSVP task, benefits or costs of the color cue conditions can clearly be attributed to direct selection by color, without mediation by location. The results showed both benefits of valid color cues and costs of invalid color cues. The authors concluded that the selective function of color is not restricted to location guidance alone but, rather, that color can be used to select items directly for further processing.

Although these results indicate that participants can use color for direct selection in an RSVP paradigm with all stimuli presented at the same location, it is unclear whether they can also use color cues when location varies. It might be that whenever a spatial component is involved, as in so much of the previously reviewed research, location dominates, and color information is used for location guidance only.

\section{Goals of the Present Study}

To investigate the dominance of location over color, it is necessary to equate the importance of location and color cues in order to avoid any bias to focus selectively on one of these features. Previous studies have generally employed these two features in different ways, preventing a comparison of the two features' effects on an equal footing (e.g., 
Humphreys, 1981; Kingstone, 1992).We therefore provided location and color cues simultaneously and with equal cue validities in a factorial design. This manipulation enabled us to assess location-cuing effects separately from color-cuing effects. In contrast to many other studies with multiple-item displays, both location and color information were additional information but were not part of the target definition. In this sense, both location and color were used in the same manner, since advance location has been used to establish the benefits of location preknowledge. In addition, the same number of alternatives was used for both cue types. The task was to discriminate whether a prespecified letter was presented in its upper- or lowercase version. A colored arrow presented at fixation point served as the cue. Both color and location cues could be valid (approximately $58 \%$ of the trials), both could be invalid (approximately 14\% of the trials), or one of the cue types could be valid while the other was invalid (approximately 14\% of the trials for each case). Eye movements were recorded to control for deviations of the eyes from fixation. The display consisted of six differentcolored letters at six different locations in a circle equally distant from the fixation point, and it was presented briefly and then masked. In this setting, valid cues should lead to a performance benefit, because the number of letters presented is too large for all to be reported (Sperling, 1960).

With location and color cues placed on an equal footing, this design allows a fair comparison of the effects of advance location and color information. Two outcomes are possible. Location might be completely dominant as a cue, in the sense that, if it is present, all other cues lose potency. In that case, the validity of the location cue should have an effect, but either valid color cues should have no effect or the effect should be mediated by location. On the other hand, it is possible that location is only one of the featuresalbeit, perhaps the most important one - that yield a cuing effect and that the effect of color is independent from that of location. In that case, color cue validity should have an effect, as well as location cue validity. A further feature of our design is that it allows us to examine possible distance effects. If selection by location is complete, performance on trials on which the items cued by color are spatially distant from the position indicated by the location cue should be worse than performance for color-cued items that are close to the cued location, because attention has to move farther in the former situation. If, on the other hand, direct selection by color is possible and independent from location information, no such distance effect should occur. Together, if color cues are effective and not mediated by location even though location is varying, performance should be better in the valid color cue condition than in the invalid color cue condition, regardless of location cue validity, and performance should not differ between color cued items close to the cued location and color cued items farther away.

\section{METHOD}

\section{Participants}

Twenty-four undergraduates (4 of them male) at the University of Otago, New Zealand, took part in this experiment. They were 18-52 years old, and their participation was part of a 1st- or 2nd-year course requirement. All of them reported normal color vision. One participant had to be replaced because performance was at chance level for all the conditions.

\section{Stimuli}

The stimuli were the upper- and lowercase versions of the letters F, $\mathrm{H}, \mathrm{R}$, and T displayed in triplex font, a built-in font of Turbo Pascal 7.0. Six of these eight different letter shapes were simultaneously presented on a dark background. They were displayed in a circular fashion $2.43^{\circ}$ from the center of the screen. All the letters were equidistant from each other and from the center of the screen. The color set consisted of six different colors; these were blue (CIE $x, y$ chromaticity coordinates of .148/.073) with a luminance of $5.32 \mathrm{~cd} / \mathrm{m}^{2}$, yellow (CIE $x, y$ chromaticity coordinates of .502/.428) with a luminance of $6.74 \mathrm{~cd} / \mathrm{m}^{2}$, red (CIE $x, y$ chromaticity coordinates of $.578 / .368$ ) with a luminance of $10.3 \mathrm{~cd} / \mathrm{m}^{2}$, green (CIE $x, y$ chromaticity coordinates of .267/.597) with a luminance of $28.0 \mathrm{~cd} / \mathrm{m}^{2}$, gray (CIE $x, y$ chromaticity coordinates of $.265 / .285$ ) with a luminance of $2.69 \mathrm{~cd} / \mathrm{m}^{2}$, and white (CIE $x, y$ chromaticity coordinates of $.265 / .290$ ) with a luminance of $36.7 \mathrm{~cd} / \mathrm{m}^{2}$. The measurements of the letters were as follows: The lowercase form of the letter $r$ had a height of $0.78^{\circ}$, whereas all other letters had heights of $1.17^{\circ}$. The widths of the letters varied from $0.49^{\circ}$ to $0.87^{\circ}$. The cue consisted of a colored arrow. This arrow pointed toward one of the possible target locations and was in one of the six colors described above. The base of each arrow was located in the center of the screen. The arrows were $0.87^{\circ}$ long and measured $0.78^{\circ}$ at the widest part. Each letter in the display was individually masked to prevent the participants from accessing visual information in iconic memory (Sperling, 1960) beyond the offset of the letter display. Thus, masks were used to limit the processing time to the presentation time of the display. The mask consisted of six layers of colored letters from the color and letter set used.

All the stimuli were viewed from a distance of approximately $59 \mathrm{~cm}$. For the target discrimination task in this experiment, the slash (“/”) key was used for right-index-finger responses and the " $z$ " key for left-index-finger responses. The assignment of the response keys to upper- or lowercase letters was counterbalanced across participants.

\section{EOG Recording}

Throughout the experiment, horizontal electrooculographic (HEOG) activity was recorded from foam tape facial electrodes placed approximately $2 \mathrm{~cm}$ lateral to the left and right canthi. To record blinks and vertical eye movements in a bipolar fashion, electrodes were attached approximately $2 \mathrm{~cm}$ above and below the midpoint of the left eye. The electrode impedances were kept under $5 \mathrm{k} \Omega$ for all electrodes, and all electrophysiological signals were filtered with a band-pass setting of $0.01-100 \mathrm{~Hz}$ and digitized at $250 \mathrm{~Hz}$. For both experimental procedures, the recording epoch for each trial began $200 \mathrm{msec}$ before the arrow cue appeared, and it lasted for a total of 3,200 msec.

\section{Procedure}

Each participant was tested in seven blocks of 84 trials in a single session lasting approximately $70 \mathrm{~min}$. The first 4 trials in each block were considered warm-up and were not recorded.

The task was to discriminate the case of a prespecified letter. A different target letter from the letter set was randomly chosen for every new block, subject to the restriction that consecutive blocks always had different target letters. The target letter information was included in the instructions presented before each block. In addition, each block began with the presentation of the capital form of this target letter in the center of the screen as a reminder. It was displayed with a height of $0.29^{\circ}$ and a width of $0.22^{\circ}$ for $500 \mathrm{msec}$.

Each trial started with a plus sign, which served as a warning signal and a fixation point. The plus sign was presented for $1,200 \mathrm{msec}$ and was followed immediately by a colored arrow cuing both location and color. The cue was presented for $150 \mathrm{msec}$. We used this short cue presentation time to avoid eye movements; such short cue 
presentation times have been shown to be effective (Cheal \& Lyon, 1991). The stimulus display appeared immediately after the offset of the cue, and it consisted of six letters arranged in a circular fashion. The display was presented for $100 \mathrm{msec}$ before a mask covered each individual letter. This display time was chosen to reduce the likelihood of eye movements and to produce a difficult but not impossible task; the same time was used by Grabbe and Pratt (2004), and it led in their experiment to an error rate of approximately $35 \%$. The fixation cross reappeared together with the letter display. The participants were informed that they should remain fixated at this point at all times. The mask remained for $300 \mathrm{msec}$, and then the screen went blank. One of the letters in each display was the target letter in either its uppercase or lowercase form. Both versions were equally likely and randomly chosen. The target appeared equally often and in a random fashion in one of the six display locations. The other letters in the display were a random mix of other letters of the letter set. No letter was presented twice per trial. Each letter was also presented randomly in a different color; accordingly, all the colors of the color set were used for each trial.

There were 84 trials in each block. In 48 of these, both the color information and the location information conveyed by the cue were valid, so the target letter appeared in the cued location and color. In 12 of these, the color cue was invalid, but the location cue was valid. In these trials, the target letter appeared in the cued location, but it was presented in a different color than had been cued; instead, one of the distractor letters was presented in the cued color. In 12 other trials, the color cue was valid, but the location cue was invalid. In these trials, the target appeared in the cued color, but in an uncued location, and a distractor letter appeared in the cued location. And for the last 12 trials, both color and location information were invalid. For these trials, the target appeared in both a different location and a different color than had been cued. Target letters appeared equally often in each of the six display positions. In invalid trials, the stimulus presented at the invalidly cued location or in the invalidly cued color was randomly chosen from the set of distractor colors and letters. Visual feedback was provided for correct and incorrect responses. In cases of errors in operation-for example, using the wrong response keys or responding before the target was shown - a visual reminder was displayed on the screen. The next trial began approximately 3,000 msec after each response.

The participants were instructed to respond as accurately as possible and to respond immediately after the detection of the target letter. They were also informed that the arrow presented at the beginning of each trial usually gave correct information about both the color and the location of the upcoming target but that sometimes one or both of these types of information would be incorrect. They were asked to use the cue information while performing the task.

Directly after the main experiment, the participants were tested in one final block, consisting of 24 trials, that was used to obtain EOG calibration data. As in the main experiment, each trial started with a 1 -sec warning signal at the center of the screen that was followed by the presentation of a colored arrow for $500 \mathrm{msec}$. After its offset, the plus sign returned at the center of the screen, and a colored letter was displayed at the cued location for $1,000 \mathrm{msec}$. The participants were instructed to focus initially on the center of the screen and then move their eyes in the direction of the arrow, to fixate briefly on the letter presented there, and subsequently to return their gaze to the fixation point. Across the 24 trials, each target position was occupied four times, twice by a capital letter and twice by a small letter. Presentation shape and location were randomized. The following trial started approximately $1,000 \mathrm{msec}$ after the letter offset.

\section{RESULTS}

For each participant separately, a standard average waveform for each display position was calculated from the EOG calibration data and was used to estimate the amount of eye movement in each individual trial. Trials with eye movements of more than $7.8 \%$ of the distance between fixation point and target were excluded. This value corresponds to eye movements of more than $0.2^{\circ}$. In total, $16.8 \%$ of all the trials were excluded. The reported results include only the remaining trials.

For the analysis of accuracy, all of the remaining trials were taken into account. RTs below $200 \mathrm{msec}$ (approximately $0.06 \%$ ) and above $2,500 \mathrm{msec}$ (approximately 1.07\%) were excluded to prevent distortion of mean RTs by a few anticipatory responses and exceptionally slow responses. All statistical tests were conducted using ANOVAs. Mean accuracies and RTs for the two levels of the two factors of color and location validity are displayed in Table 1 .

\section{Analysis of Accuracy}

An overall ANOVA on the percentages of correct responses included the following within-subjects factors: location validity (valid vs. invalid), color validity (valid vs. invalid), target letter case (upper vs. lower), and practice (first three blocks vs. last four blocks). Two planned comparisons were also conducted to evaluate the effect of color validity separately for the conditions with valid location cues and with invalid location cues. In addition, two more ANOVAs were conducted to examine distance effects. In one, the within-subjects factors of distance (far vs. near) and validity (location cues invalid/color cues valid vs. both cue types invalid) were included. For the second ANOVA, the same factors were used, but with the inclusion of the validity condition with valid location cues and invalid color cues as a third level of the validity factor.

In the overall ANOVA, the effect of validity for location cues amounted to a $26 \%$ advantage of valid location cues over invalid location cues and was, as was expected, highly significant $\left[F(1,23)=101.02, M S_{\mathrm{e}}=645.96, p<.001\right]$. Moreover, the $5.2 \%$ advantage of valid color cues over invalid color cues was also significant $[F(1,23)=15.53$, $\left.M S_{\mathrm{e}}=169.22, p<.002\right]$. The interaction between the factors of location and color validity did not reach significance ( $p=.257)$. In the planned comparison including only the conditions with valid location cues, there was a significant accuracy benefit of $4.3 \%$ for trials with valid location and color cues over trials in which color was invalid but location was valid $\left[F(1,23)=16.25, M S_{\mathrm{e}}=53.16, p<.002\right]$. Also significant was the $6.2 \%$ performance benefit of color for trials in which color cues were valid but location cues were not, as compared with the trials in which both cue types were invalid $\left[F(1,23)=10.01, M S_{\mathrm{e}}=185.42, p<.005\right]$.

The overall analysis of accuracy also revealed one other significant result. There was an interaction of target letter case with color cue validity $\left[F(1,23)=8.35, M S_{\mathrm{e}}=\right.$ $64.39, p<.01]$. Accuracy for lowercase target letters remained relatively stable in valid and invalid color cue trials, whereas for uppercase target letters, performance was better in valid color cue trials than in invalid color cue trials. This result can be explained by a tendency to guess more often in favor of lowercase letters, which has been observed before (Vierck \& Miller, 2005). There was no practice effect for the accuracy data. ${ }^{1}$

In a further analysis, we examined distance effects. One way to process the two cue types is in a serial manner; that 
is, the participants would first attend to the cued location, and if this location was invalid, they would then attend to the item in the cued color. If the selection of the colored item is mediated by location, items in the cued color that are spatially farther away from the position indicated by the location cue should be identified less accurately than items that are close to the cued position, because attention has to travel farther in a limited time frame, making these trials more difficult. To examine this possibility, we used trials with invalid location trials only. For these trials, we compared performance for all trials in which location and color cues led to positions close to each other with performance for trials in which these cues led to positions farther away. For the purpose of these distance analyses, the two locations directly adjacent to the cued location were defined as close, whereas all the other locations in the display were defined as distant. The results are displayed in Figure 1. No significant main effect for the distance factor was found ( $p=.951)$, but the interaction between distance and validity was significant $\left[F(1,23)=6.65, M S_{\mathrm{e}}=\right.$ $0.01, p<.02]$. For trials in which color cues were valid and location cues were invalid, performance was better by $4.2 \%$ when the color cued item was close to the position cued by the location cue than when it was farther away. This result indicates that the participants first selected the cued location and, when this was found to be invalid, shifted to the location containing the cued color. For trials in which both cue types were invalid, performance was $4 \%$ worse for color cued items closer to the cued location than for color cued items farther away. Thus, invalid color cues hurt performance when they led to a position close to the cued location. This again indicates that participants select primarily by location. In trials with valid location cues, however, there was no effect of the distance to the color cue $(p=.789)$, suggesting that attention was not shifted when the location cue was valid.

We also investigated whether performance on trials with invalid location cues decreased with a larger distance between the cued location and the target position. Given that performance for trials with validly cued locations was much better in general than that for invalidly cued locations, one would expect that for location cues that point toward a position farther away from the target position, performance would be impaired, because attention would have to travel a longer distance to the target position than for nearby target positions. For this analysis, we used only trials in which location cues were invalid, and we compared trials in which color cues were valid with trials in which both cue types were invalid. As was expected, we found a significant advantage for target letters presented close to the cued location $\left[F(1,23)=5.67, M S_{\mathrm{e}}=0.01\right.$, $p<.05]$. This distance effect confirms the effectiveness of location information.

\section{Analysis of RT}

Although the main dependent measure was response accuracy, analyses of RT will also be reported for completeness, although we acknowledge that the interpretation of $\mathrm{RT}$ as a measure of the time needed to perform the task is complicated by the high error rates. Indeed, mean RT over
Table 1

Average Percentages Correct (PCs) and Reaction Times

(RTs, in Milliseconds) As a Function of the Validity of the Location Cue and the Color Cue

\begin{tabular}{cccccc}
\hline & \multicolumn{3}{c}{ Location Cue } \\
\cline { 2 - 3 } \cline { 5 - 6 } Color Cue & PC & RT & & PC & RT \\
\cline { 2 - 5 } \cline { 4 - 6 } Valid & 802 & & 67.4 & 1,082 \\
Invalid & 92.5 & 878 & & 61.2 & 1,133 \\
\hline
\end{tabular}

all conditions was $974 \mathrm{msec}$, which is rather long for a twochoice discrimination task. Henderson (1991), for example, reported mean RTs of $669 \mathrm{msec}$ in a similar design, albeit with stimulus conditions producing approximately $87 \%$ correct responses overall. Given that our stimulus conditions were chosen to produce higher error rates, it is not surprising that RTs were also longer in our experiment.

In general, the pattern for RTs was similar to that observed for accuracy. The effect of location cue validity was also highly significant for RT $\left[F(1,23)=68.43, M S_{\mathrm{e}}=\right.$ $100,537.2, p<.001]$, with an advantage of $267 \mathrm{msec}$ for valid location cues over invalid location cues. Also, the effect of color cue validity was significant, with a 63-msec advantage of valid color cues over invalid color cues $\left[F(1,23)=19.92, M S_{\mathrm{e}}=19,363.5, p<.001\right]$. The interaction between location and color validity did not reach significance ( $p=.104)$. The additional comparison with only valid location cues yielded a significant benefit of $76 \mathrm{msec}$ for both-cues-valid trials, as compared with trials in which color was invalid and location was valid $\left[F(1,23)=23.0, M S_{\mathrm{e}}=11,999.3, p<.001\right]$. The additional analysis with only location-invalid trials also showed a reliable advantage of $51 \mathrm{msec}$ for trials in which color was valid but location was not, as compared with trials in which both cue types were invalid $[F(1,23)=9.92$, $\left.M S_{\mathrm{e}}=12,552.2, p<.005\right]$.

The analysis of RT also showed a practice effect $\left[F(1,23)=78.27, M S_{\mathrm{e}}=38,844.8, p<.001\right]$, with RT decreasing over blocks. This practice effect was larger for invalid color cue trials than for valid color cue trials $\left[F(1,23)=4.39, M S_{\mathrm{e}}=7,909.2, p<.05\right]$. There was also a three-way interaction involving the factors of color validity, location validity, and blocks $\left[F(1,23)=5.03, M S_{\mathrm{e}}=\right.$

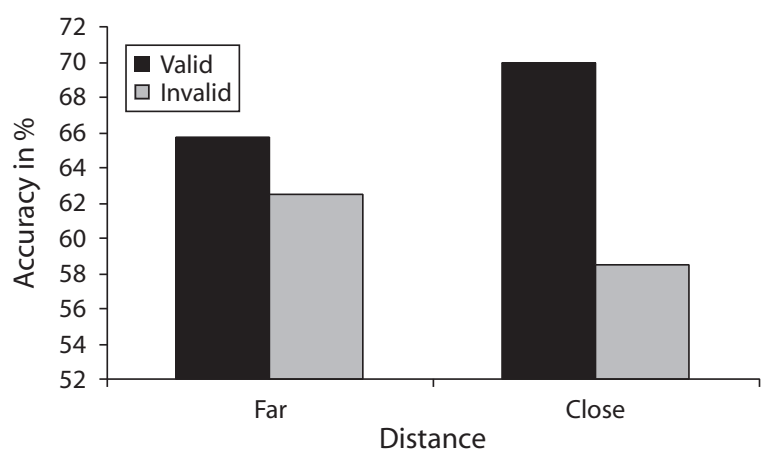

Figure 1. Distance effects in accuracy for trials on which location was invalid when color cues were either valid or invalid. 
$3,825.0, p<.05]$. In the first few blocks, valid color cues led to a larger RT benefit when location cues were valid than when they were invalid. For later blocks, this advantage disappeared, leading to a similar color-cuing effect, regardless of location validity. This suggests that the larger practice effect observed for invalid color cues was due mainly to comparatively long RTs for invalid color cue trials at validly cued locations. In addition, responses to uppercase target letters were faster than responses to lowercase target letters $\left[F(1,23)=14.47, M S_{\mathrm{e}}=9,312.0\right.$, $p<.002]$. This factor interacted with validity for location $\left[F(1,23)=8.55, M S_{\mathrm{e}}=3,589.7, p<.02\right]$. For valid location cues, responses to lowercase target letters were only slightly slower than those to uppercase target letters; this difference was much larger for invalid location cue trials. Thus, it seems that the larger uppercase letters enabled faster responses and that this advantage was more pronounced for invalid location cue trials.

As for accuracy data, further analyses were conducted to examine distance effects. None of the effects observed in the analysis of accuracy was significant in the analysis of RT.

\section{DISCUSSION}

In an experiment that equated advance location and color information, we demonstrated the usefulness of advance location information, as have many other studies before (e.g., Cheal \& Gregory, 1997; Posner et al., 1980). Valid location cues led to much better performance (i.e., mainly higher accuracy, but also faster responding) than did invalid location cues. In addition, the difference in size between the location-cuing effect and the color-cuing effect supports a dominant role of location in the selection process (e.g., Nissen, 1985; Tsal \& Lavie, 1993). Although color cues were effective, further analyses of distance effects indicate that this benefit was mediated by location. The participants seem to have first selected the cued location and then, if necessary, shifted attention to the location of the item in the cued color. Together, these findings suggest that location is indeed special, in that although direct selection by color is possible in single-location displays (Vierck \& Miller, 2005, 2006), it is not used in a multiple-item display when location varies. Note, however, that attention to location seems to be influenced by color cues as well as location cues, as indicated by the finding that performance was better when both cues were valid than when just the location cue was valid. This finding suggests that attention is more fully focused on the target item when the location and color cues agree as to the location of that item than when they disagree.

This finding confirms and extends previous findings (e.g., Nissen, 1985; Tsal \& Lavie, 1993). For example, in Nissen's experiment, location information was integral for all the conditions, which might have biased the participants to focus on location information. Because, in our experiment, location and color information were equally helpful, we can be sure that such a bias did not occur. In addition, the cued features in Nissen's experiment had to be used in order to fulfill task requirements. We employed location and color information within a typical cuing paradigm, where information is only additional and, thus, indepen- dent from task requirements per se. This feature allowed a more specific evaluation of the selection process.

The color-cuing effect observed when both cue types were valid, as compared with trials in which only the location cue was valid, seems to confirm the effect described by Humphreys (1981). He, as well, found that color preknowledge improved performance even when the target location was known. His result is difficult to explain under the assumption of complete location dominance, because location information was always valid and, therefore, led the participants to the correct item. This should have been sufficient for optimal performance if attention is guided entirely by location, but valid color information improved performance beyond the level observed with only valid location cues. In Humphreys's experiment, color may have been effective only because the locations were rather confusable. In contrast, the stimuli in our experiment were much farther apart, with adjacent stimuli separated by approximately $2.2^{\circ}$. This enabled us to avoid potential effects of location confusability and also to analyze distance effects to investigate attentional shifting. The analysis of distance effects was based on the widely accepted but not uncontroversial idea (Yantis, 1988) that attention travels with constant velocity through space. Our finding that accuracy decreases with increasing distance is compatible with some previous results suggesting improved performance for targets in adjacent locations (e.g., Hoffman \& Nelson, 1981) but is incompatible with other cases in which accuracy seemed to increase with increasing distance (e.g., Bahcall \& Kowler, 1999; Cutzu \& Tsotsos, 2003).

The fact that the participants had enough time to shift covert attention from the cued location to potential target locations close by indicates that attentional shifts are very fast. In our experiment, the $100-\mathrm{msec}$ display presentation was rather short. Reported times needed for attentional shifts vary widely. For example, Saarinen and Julesz (1991) estimated that under optimal conditions, attentional shifts could occur within $50 \mathrm{msec}$, but this time increased by at least $160 \mathrm{msec}$ in situations in which a cued item had to be identified before attention could shift (Kröse \& Julesz, 1989). Wolfe, Alvarez, and Horowitz (2000) estimated that $200 \mathrm{msec}$ per item were needed to search a display similar to the one used here, although this includes the time needed for item examination time, as well as for shifting attention. Others have found that it takes approximately $300 \mathrm{msec}$ to shift attention fully to a new location (Cheal \& Lyon, 1991). Overall, these findings indicate that the participants had, at most, enough time to shift once to a position close by. Another possibility is that they covertly attended to the cued location without moving their eyes when the cue came on. The fact that performance suffered when location cues were invalid and the validly cued color location was far away from the cued location suggests that presentation time was insufficient for an attentional shift to a place farther away.

Although the present experiment indicates that selection by color in multiple-item displays is mediated by location, it is interesting to consider the possibility that the overall performance levels represent a mixture of some trials on which they attended primarily to location cues and other 
trials on which they attended primarily to color cues. One possibility, for example, is that some participants attended mostly to the cued locations, whereas other participants attended mostly to the cued colors. If so, there should have been a negative correlation, across participants, between the sizes of the location-cuing effect and the color-cuing effect. Participants who attended mostly to location cues should perform much worse when a location cue is invalid but should be relatively unaffected by an invalid color cue. In contrast, participants who attended mostly to color cues should show exactly the reverse pattern. To test this idea, we computed the correlation across participants in the sizes of these two effects for the accuracy data. The observed correlation was $r=-.40$, which was not quite significantly different from zero $(p>.05)$ but showed a clear trend $(p<.10)$. Closer inspection of the results, however, revealed that they were strongly influenced by 4 participants who seemed to have focused entirely on location cues. As a consequence, they had very low accuracy in the condition with valid color cues but invalid location cues, even lower than in the condition in which both cue types were invalid, and very accurate performance on both trial types with valid location cues. Without these participants, color-cuing effects appear to be independent of location-cuing effects. This would suggest that although advance location information is the preferred selection feature for most people most of the time, selection can potentially occur directly by color even when location varies. This possibility is in agreement with the Boolean map theory recently proposed by Huang and Pashler (2007; see also Huang, Treisman, \& Pashler, 2007). According to this theory, participants can choose either to select one feature of one dimension or to select multiple locations at the same time. If a feature is chosen, the feature value (e.g., color) and locations of this feature can be consciously accessed. On the other hand, if multiple spatial locations are selected, these locations can be accessed, but not the feature values at these locations.

In conclusion, whether selection by color is direct or mediated by location seems to depend on the layout of the experiment. Vierck and Miller $(2005,2006)$ found clear evidence of direct selection by color. Both experiments were almost identical, apart from the presentation of all the stimuli simultaneously in a spatial design in the present experiment and the presentation of the stimuli within an RSVP design - that is, one item at a time in our previous experiments. In sum, it seems that although direct selection by color is possible, in spatial designs with multiple stimuli locations, selection by location is the preferred option.

\section{AUTHOR NOTE}

This research was supported in part by a Marsden grant from the Royal Society of New Zealand to J.M. We thank Kyle Cave, Jan Theeuwes, Dominique Lamy, and four anonymous reviewers for helpful comments on earlier versions. Correspondence concerning this article should be addressed to E. Vierck or J. Miller, Department of Psychology, University of Otago, Dunedin, New Zealand (e-mail: esther@psy.otago.ac.nz or miller@psy.otago.ac.nz).

\section{REFERENCES}

BahCALl, D. O., \& Kowler, E. (1999). Attentional interference at small spatial separations. Vision Research, 39, 71-86.
Brawn, P., \& Snowden, R. J. (1999). Can one pay attention to a particular color? Perception \& Psychophysics, 61, 860-873.

Cave, K. R., \& Pashler, H. (1995). Visual selection mediated by location: Selecting successive visual objects. Perception \& Psychophysics, 57, 421-432.

Cheal, M., \& Gregory, M. (1997). Evidence of limited capacity and noise reduction with single-element displays in the location-cuing paradigm. Journal of Experimental Psychology: Human Perception \& Performance, 23, 51-71.

Cheal, M., \& Lyon, D. R. (1991). Central and peripheral precuing of forced-choice discrimination. Quarterly Journal of Experimental Psychology, 43A, 859-880.

Cutzu, F., \& Tsotsos, J. K. (2003). The selective tuning model of attention: Psychophysical evidence for a suppressive annulus around an attended item. Vision Research, 43, 205-219.

Egeth, H. E., Virzi, R. A., \& Garbart, H. (1984). Searching for conjunctively defined targets. Journal of Experimental Psychology: Human Perception \& Performance, 10, 32-39.

Garner, W. R. (1987). Location and color as cuing dimensions in contingent classification. Perception \& Psychophysics, 41, 202-210.

Gold, J. M., \& PratT, J. (2001). Is position "special" in visual attention? Evidence that top-down processes guide visual selection. Canadian Journal of Experimental Psychology, 55, 261-270.

Grabbe, Y., \& Pratt, J. (2004). Competing top-down processes in visual selection: Evidence that selection by location is stronger than selection by color. Journal of General Psychology, 131, 137-149.

Henderson, J. M. (1991). Stimulus discrimination following covert attentional orienting to an exogenous cue. Journal of Experimental Psychology: Human Perception \& Performance, 17, 91-106.

Hoffman, J. E., \& Nelson, B. (1981). Spatial selectivity in visual search. Perception \& Psychophysics, 30, 283-290.

Huang, L., \& Pashler, H. (2007). A Boolean map theory of visual attention. Psychological Review, 114, 599-631.

Huang, L., Treisman, A. M., \& Pashler, H. (2007). Characterizing the limits of human visual awareness. Science, 317, 823-825.

HÜBNER, R., \& BACKER, G. (1999). Perceiving spatially inseparable objects: Evidence for feature-based object selection not mediated by location. Journal of Experimental Psychology: Human Perception \& Performance, 25, 1556-1567.

Humphreys, G. W. (1981). Flexibility of attention between stimulus dimensions. Perception \& Psychophysics, 30, 291-302.

Kaptein, N. A., Theeuwes, J., \& Van Der Heijden, A. H. C. (1995), Search for a conjunctively defined target can be selectively limited to a color-defined subset of elements. Journal of Experimental Psychology: Human Perception \& Performance, 21, 1053-1069.

KIM, M.-S., \& CAVE, K. R. (2001). Perceptual grouping via spatial selection in a focused-attention task. Vision Research, 41, 611-624.

Kingstone, A. (1992). Combining expectancies. Quarterly Journal of Experimental Psychology, 44A, 69-104.

Kröse, B. J., \& Julesz, B. (1989). The control and speed of shifts of attention. Vision Research, 29, 1607-1619.

Lambert, A. J., \& Corban, R. (1992). Spatial attention and expectancy for color, category and location: Further evidence against the spotlight model. Acta Psychologica, 81, 39-51.

LAmY, D., \& TsaL, Y. (2000). Object features, object locations, and object files: Which does selective attention activate and when? Journal of Experimental Psychology: Human Perception \& Performance, 26, $1387-1400$.

Monheit, M. A., \& Johnston, J. C. (1994). Spatial attention to arrays of multidimensional objects. Journal of Experimental Psychology: Human Perception \& Performance, 20, 691-708.

Moore, C. M., \& Egeth, H. (1998). How does feature-based attention affect visual processing? Journal of Experimental Psychology: Human Perception \& Performance, 24, 1296-1310.

NisSEN, M. J. (1985). Accessing features and objects: Is location special? In M. I. Posner \& O. S. M. Marin (Eds.), Attention and performance XI: Mechanisms of attention (pp. 205-219). Hillsdale, NJ: Erlbaum.

Poisson, M. E., \& WiLkinson, F. (1992). Distractor ratio and grouping processes in visual conjunction search. Perception, 21, 21-38.

Posner, M. I., Snyder, C. R. R., \& Davidson, B. J. (1980). Attention and the detection of signals. Journal of Experimental Psychology: General, 109, 160-174.

SaArinen, J., \& Julesz, B. (1991). The speed of attentional shifts in 
the visual field. Proceedings of the National Academy of Sciences, 88, 1812-1814.

Shen, J., Reingold, E. M., \& Pomplun, M. (2000). Distractor ratio influences patterns of eye movements during visual search. Perception, 29, 241-250.

SHIH, S.-I., \& SPERLING, G. (1996). Is there feature-based attentional selection in visual search? Journal of Experimental Psychology: Human Perception \& Performance, 22, 758-779.

Sobel, K. V., \& CAVE, K. R. (2002). Roles of salience and strategy in conjunction search. Journal of Experimental Psychology: Human Perception \& Performance, 28, 1055-1070.

SPERLING, G. (1960). The information available in brief visual presentations. Psychological Monographs: General \& Applied, 74, 1-29.

THEEUwes, J. (1989). Effects of location and form cuing on the allocation of attention in the visual field. Acta Psychologica, 72, 177-192.

Tsal, Y., \& Lamy, D. (2000). Attending to an object's color entails attending to its location: Support for location-special views of visual attention. Perception \& Psychophysics, 62, 960-968.

Tsal, Y., \& Lavie, N. (1988). Attending to color and shape: The special role of location in selective visual processing. Perception \& Psychophysics, 44, 15-21.

TsaL, Y., \& LAVIE, N. (1993). Location dominance in attending to color and shape. Journal of Experimental Psychology: Human Perception \& Performance, 19, 131-139.

Vierck, E., \& Miller, J. (2005). Direct selection of color for visual encoding. Perception \& Psychophysics, 67, 483-494.

ViercK, E., \& Miller, J. (2006). Effects of task factors on selection by color in the rapid serial visual presentation (RSVP) task. Perception \& Psychophysics, 68, 1324-1337.

Wolfe, J. M., Alvarez, G. A., \& Horowitz, T. S. (2000). Attention is fast but volition is slow. Nature, 406, 691 .

YANTIS, S. (1988). On analog movements of visual attention. Perception \& Psychophysics, 43, 203-206.

Zohary, E., \& Hochstein, S. (1989). How serial is serial processing in vision? Perception, 18, 191-200.

\section{NOTE}

1. Essentially the same results were obtained in an analysis of behavioral results from all the trials - that is, one including those trials in which eye movements had occurred.

(Manuscript received March 22, 2007; revision accepted for publication September 11, 2007.) 